\title{
REPAIR OF MISMATCHED BASEPAIRS IN MAMMALIAN DNA
}

PROGRESS REPORT

FOR FINAL FUNDING YEAR

3/1/90 TO 2/28/91 (no cost extension to 5/31/91)
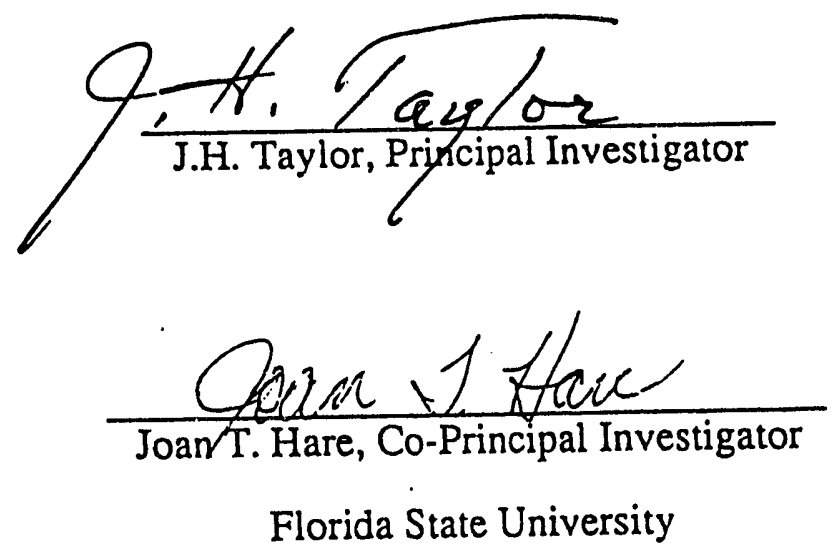

Institute of Molecular Biophysics

Tallahassee, Florida 32306

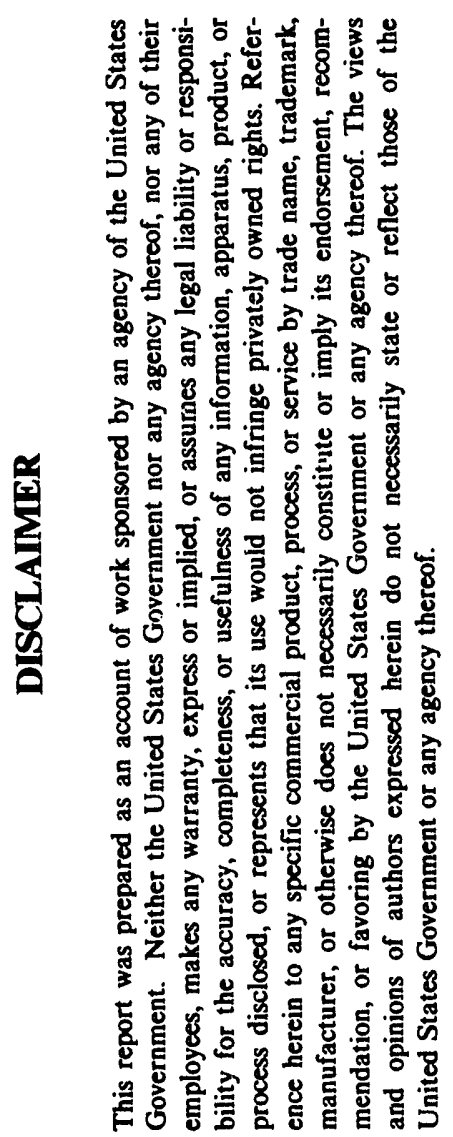

AUGUST, 1991 
OVERVIEW OF OUR EFFORT

For the past eight years DOE has supported our work in DNA mismatch repair. During that period we developed a transfection system for assaying the in vivo repair of specific DNA mismatches in mammalian cells. We have used that system to study factors which can influence the choice of template strands in the mismatch repair event. For an accounting of all our earlier results, please see previous Progress Reports.

During our final year of funding we have concentrated on three specific areas of our originally proposed research plan. As some of our reviewers recommended, we put our greatest emphasis on extending our work on the role of single strand nicks in influencing template strand selection in mismatch repair. This area appeared to be the most likely to produce useful results. Our results in this area over the last several months have been exciting. We found, that the ability of a nick in one strand to influence which strand is repaired is not a simple function of distance from the mismatched site. We identified one "hot spot" where a nick is more likely to have an influence. A manuscript of this work is currently in preparation, with submission planned within the month.

The second line of effort was the production of single-genotype heteroduplexes in order to examine independently the repair of $T / G$ and $A / C$ mispairs within the same sequence context as in our mixed mispair preparations. After many months work in previous granting periods, a variation of one of the several approaches we had tried was finally made to work. In March of this year we proved that we could produce preparations of supercoiled heteroduplex that were exclusively $\mathrm{T} / \mathrm{G}$ or exclusively $\mathrm{A} / \mathrm{C}$ at the mispair site. This work was presented as a short report at the 1991 Miami Winter Symposium. Subsequently, the editors of two journals have invited us to submit this work for publication in their respective journal. I expect to submit portions of this work as two separate papers to Methods in Molecular and Cellular Biology and

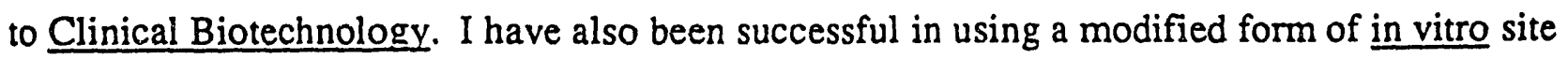
directed mutagenesis to produce two new mutant SV40 DNA's in the last year that will allow us to examine mismatch repair of the same mispairs, but in a new site in the genome. 
The third area of research effort has been in trying to understand the difference in repair bias of different cell lines or different transfection conditions as it may relate to different repair systems in the cell. We have identified some of the sources of variation, including cell cycle position. We hope to continue this work to more precisely identify the phase of the cell cycle. Our results in this area lay the foundation for future work.

\section{SPECIFIC RESULTS}

\section{The Effect of Nicks on Template Strand Choice}

Our method of producing heteroduplexes in SV40 DNA involves digesting each of two genotypes of DNA with a different restriction enzyme which cuts at a unique site in the circular DNA. Denaturation of the mixed linear DNA's, followed by reannealing of the single strands results in heteroduplexes which form circles due to their long complementary sticky arms. The circular form of the heteroduplexes, but not the homoduplexes, allows their separation on a gel. The two sites of restriction used in construction are nicks in the final heteroduplex (Figure 1). The position of the nicks can be varied by choice of unique restriction sites in the starting DNA's. Moreover, one can reverse which starting DNA is cut with which enzyme.

In earlier work we demonstrated that we $\underset{F}{\mathcal{C}}$ ould alter the bias of the repair of a heteroduplex by switching which strand carried the nick. The strand with the nick was much more likely to be removed during repair. We have now expanded this work to determine if the ability of the nick to influence diminishes over distance.

We used the technique outlined above to construct a large number of pairs of heteroduplexes. A pair consists of one heteroduplex in which DNA 1 has been cut at enzyme site $A$ and DNA 2 has been cut at enzyme site $B$ and a second heteroduplex in which DNA 2 has been cut at enzyme site A and DNA 1 has been cut at enzyme site B. By comparing the percentage of repair products with the genotype of DNA 1 versus DNA 2 produced following the transfection with one or the other of the pair of heteroduplexes, we can see the degree of effect of a particular nick site. (Our last report outlines our method of PCR plus restriction analysis to determine genotype of repaired DNA.) One of the nick sites in each pair was held 


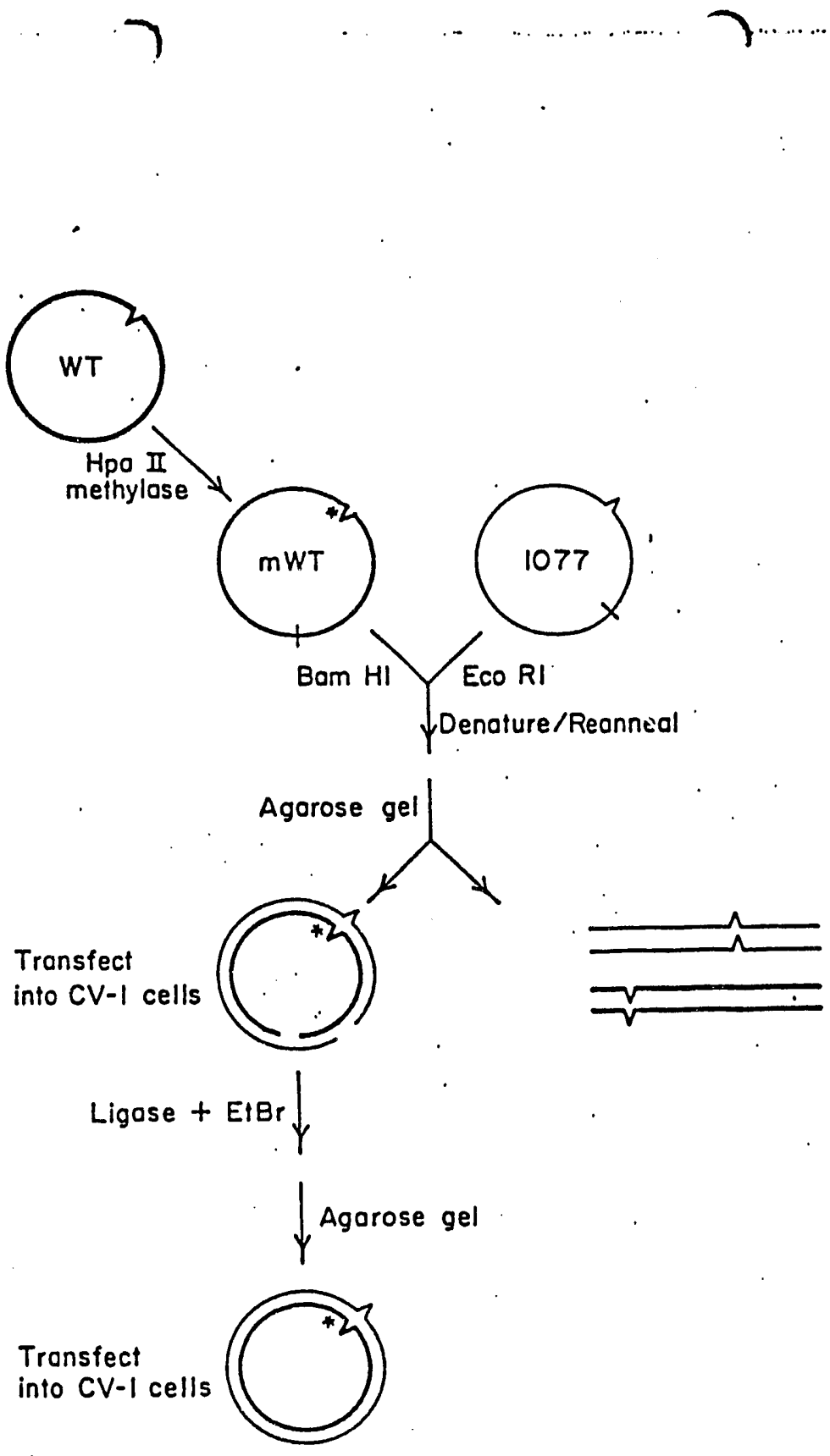

FIGURE 1

Figure 1 - Method of construction of heteroduplexes. Two SV40 DNA's which differ at a single base pair in the unique HpaII site are linearized by restriction at unique but different restriction sites. One or both parents may be methylated with commercially available methylases. The linearized DNA's are mixed, then denatured and reannealed. Any strands of the same genotype which come together during reannealing will be linear, but when strands of different genotype (therefore different sites of linearization) come together they will form long sticky arms which can then reanneal to form a circle. Linear and circular DNA is separated by gel

electrophoresis. The nicks of the circular DNA can then be closed with $T_{4}$ DNA ligase. Intercalation of ethidium bromide into the covalently closed circles allows separation of open circles from covalently closed circles. 
constant among all heteroduplex pairs and was the maximum possible distance from the mispair (2180 bp out of 5243). The second nick was placed at varying distances from the mispair to test the effect of distance of the nick from the mispair on the choice of which strand is repaired. Eleven unique restriction sites were used ranging from 50 bases to more than 1,400 bases from the mispair. These sites represent an exhaustive study of the unique restriction sites in SV40 (with the exception of one enzyme not commercially available). The results of this work which represent the analysis of well over 1,000 individual plaques is shown in Figure 2.

No strong dependence on distance was revealed in the results of these transfections. Instead, a striking hot spot was discovered almosi 700 bases from the mispair whose effect was almost 3 times greater than its nearer neighbor at 500 bases from the mispair. By contrast, two other sites showed very little ability to influence strand selection though their neighboring sites showed effects.

That the second (distant) nick in each heteroduplex had little effect on repair was demonstrated by the repair results from a set of heteroduplexes in which the second nick was placed in positions closer to the first nick. The idea tested was whether there was a competition between the two nicks in a single heteroduplex. Competition should be visible as a diminution of the influence of the closer nick as the second nick is moved closer to it. However, in every case the nick nearer to the mispair had the same degree of effect regardless of position of the second nick (as long as the second nick was further away). Although the data is not shown the conslusions are drawn from the analysis of over 500 individual plaques.

We attempted to correlate "hot spots" to known gene regions. While the primary hot spot was approximately 100 bases 3' to the start site of the Vp3 gene, another site approximately 130 bases 3' to the start of the Vp1 gene had a much more modest effect when nicked. A nick within the origin of replication was no more influential than several other equi-distant or more distant sites.

We also examined $\mathrm{G}+\mathrm{C}$ content of short regions around the restriction sites and found no correlation between the degree of influence and local sequence. 


\section{Effect of distance of nick from mispair on ability to influence repair blas}

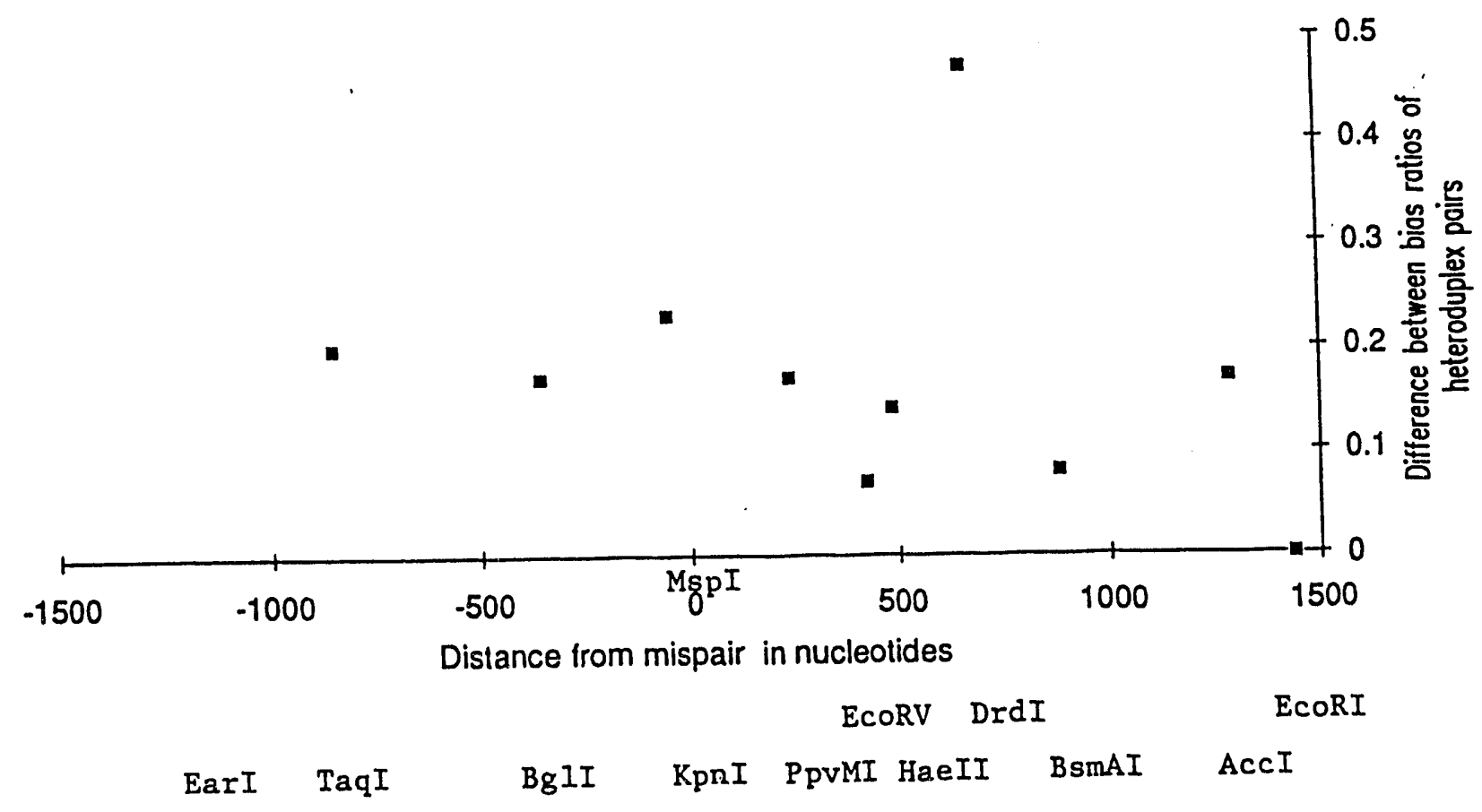

FIGURE 2 - SV40 heteroduplexes were formed by reannealing denatured DNA's of two genotypes which differed at only one base pair. Each heteroduplex canied two separate nicks which were on opposite strands. A heteroduplex pair was constituted of the two alternative pairings of nick to DNA genotype. CV-1 (African Green Monkey kidney) cells were transfected with either of the members of the heteroduplex pair and the resulting progeny plaques were analyzed by PCR plus restriction digest to determine which genotype it contained. Bias ratio was the number of plaques of one genotype divided by the total number of plaques of a single genotype produced by that heteroduplex. The difference between the bias ratios of a pair of nicked heteroduplexes is equal to the proportion of plaques repaired to $A: T$ when the closer nick was in the $C: G$ genotype strand minus the proportion of plaques repaired to A:T when the closer nick was in the A:T genotype strand. This difference is an indicator of whether a particular nick has an effect on the repair bias. If switching the nick to the strand with the alternate genotype has an effect on the repair bias. If switching the nick to the strand we the
does not alter the bias ratio, then the nick is presumed to have no effect. Alternatively, any
positive difference in the bias ratio indicates an effect from the nick. A positive difference indicates that the closer nick tended to increase the removal of the strand it was in. 
We have considered the possibility that the hot spot might correlate with secondary replication origins or preferred sites for Okazawki fragment initiation.

Presumably the nicks in these constructions act as entry sites for exonucleases which remove the nicked strand. For this reason we are currently testing the ability of exonucleases to recognize and act on nicks at these specific sites.

In future work I would like to examine whether the entire strand between the nick and the mispair is removed or whether the position of the nick is communicated to repair proteins acting only at the site of the mispair. One approach to determine this is to radioactively label at ' high specific activity the 3 ' end at the nick site, overload the host cells with the labeled heteroduplex and recover transfecting DNA after 48 hours and examine its genotype. Loss of label in a restriction pattern indicative of repair and retention of the label in unrepaired molecules would indicate that repair involved the removal of the region between the nick and mispair on the nicked strand.

\section{Production of New Constructions to Test New Repair Sites}

Will these sites which act as hot spots when nicked also be hyperactive for mismatches in other regions of the genome? This question can now be addressed using a new pair of mutints we have just produced which create a new restriction enzyme site while destroying an existing site in the body of the Vp1 gene. These mutants will allow me to create a heteroduplex with a single mispair at a new site in the genome while retaining the same local sequence context as at the previously tested mispair site. We have theorized that the position of a mispair within the genome may influence the type of repair system used to correct it. Different repair systems probably correct mispairs with different biases. The particular restriction enzyme site chosen for mutagenesis and the new overlapping restriction enzyme site created allows a restriction analysis of the progeny of the transfected heteroduplexes to reveal how the mispair was repaired.

In the original mispair, for which we have a considerable body of data, the unique Hpall site from WT was mispaired to a complementary sequence of a mutant which, by the change of a single base, produced a PvuII site at the same site. Restriction analysis of the progeny produced when these heteroduplexes were transfected into host cells, revealed which sequence was 
retained by the repair. In selecting a new site, one of the three additional PvuII sites in the genome was selected as a target for mutagenesis. The original mispair was in a promoter region of the viral late genes; the new site chosen is within the body of the viral late genes. By altering one base immediately adjacent to the PvuII site in the Vpl gene, we could set up an identical situation to our first mispair, in that when paired with a mutant produced by an additional change of a single base, could produce either a Hpall site or a Pvull site upon repair of the single mispair.

To oroduce these mutants, I used one of two oligonucleotides bearing the desired changes and performed PCR (polymerase chain reaction) using a second primer which primed in the opposite direction from the cut site of a unique restriction site. The product of this reaction, an approximately 1,400 base pair segment (which could not be simply inserted into the remainder of the SV40 molecule because the mutant oligonucleotide was not part of a unique restriction site) was then used as a mega primer to complete extension on a second template. The second template was full length SV40 cut at the unique restriction site used in the second primer in the first PCR. Of the double strand of the mega primer, only one strand could be extended on the template in the second polymerase reaction. When annealed to the second template, the 5 ' end of one of the strands of the mega primer produced the appropriate 5 ' overhang which would result from digestion with the unique restriction enzyme. The 3' end of this mega primer was the complement of the original mutant oligonucleotide. The long 5 ' extension in front of the mutant site in the mega primer protected it from being displaced by the polymerase. A higher ratio of mutant mega primer to second template assured that upon denaturation and reannealing, the predominant double stranded product would be the mega primer reannealed to the template. (However, when the mutant mega primer is present at too high a ratio the polymerase acts predominantly on it, forming artifacts.) Following extension on the second template, this product was ligated. The product of this reaction was transfected into host monkey cells and resultant plaques were isolated. Because plaques can be isolated from SV40 transfections, subcloning in a single stranded bacteriophage such as M13 is not necessary. 
In mutagenesis with one of the two oligonucleotides, restriction analysis of 24 of these plaques revealed that eight produced the restriction pattern expected of the desired mutant.

These mutant DNA's will now be used to construct heteroduplexes and bias of their repair will be compared to that of the mispair in a different genomic context. The comparison will first be of heteroduplexes with no free ends, i.e., supercoiled. We will then test heteroduplexes containing nicks to see if the same nick sites have the same effect on a mispair in this region.

\section{Production of New Constructions to Separately Analyze Repair of G/T and A/C}

\section{Mismatches}

A continuing goal of our research has been to produce and test the $G / T$ and $A / C$ mismatches separately. All previous work has been with a statistically 50:50 mix of the $\mathrm{G} / \mathrm{T}$ and A/C. While we have demonstrated such mixes can provide useful information, the ideal experiment would test only one of the mispairs at a time. We will now be able to do just that. In the production of these separate mispairs we have had to overcome several obstacles. One of the major obstacles was that our final product had to contain no incomplete products or by-products. Also, we felt strongly that we wanted to produce the separate mispairs at the mispair site for which we had already accumulated so much data. Although separate $G / T$ and $A / C$ containing heteroduplexes of SV 40 have been produced by other workers, they took advantage of a pair of adjacent unique restriction sites to insert a unidirectional synthetic DNA insert. The use of this method is restricted to sites where two restriction sites are adjacent and presumably within a non-essential region. There is only one such site available in SV40.

Although, we had spent some effort in past granting periods attempting to insert mismatch containing oligonucleotide cartridges into a unique restriction site which we engineered into a noncoding region of SV40, we met with only limited success in producing supercoiled DNA's in these particular constructs (we felt it was a requirement to prove there were no nicks remaining in the heteroduplex - especially so near the mispair). Also, the limited number of plaques we recovered from transfection with these DNA's indicated insertions were 
not tolerated well at this site, even though it was an intragenic site. Even when these efforts were successful, we still thought it imperative to return to the original mispair site.

The preparative scale separation of single strands of SV40 DNA is required to produce these single genotype mismatches. The size of SV40 makes it a poor candidate for the production of single stranded DNA in a single stranded phage vector, therefore, we chose a polymerization method to produce the specific single strands.

Ultimately, we constructed a gapped duplex molecule of SV40 using a single unique restriction site in one DNA and two different unique sites which flanked the mispair site in the second DNA. Denaturation of the full length DNA and the large fragment of the second DNA, followed by their reannealing produced a gapped circle which was separated from the starting material by virtue of its differing mobility on an agarose gel. Furthermore, the gapped circle could be separated on the gel from any contaminating full circle. The gap was filled by either of two asymmetric PCR products that were produced from a single primer that reproduced one of the restriction sites, plus a template that was cut at the second restriction site. Consequently, PCR produced specific single strands with the exact ends necessary to fill the gap. That we had produced the appropriate single strand products was demonstrated by their mobility on an acrylamide gel. We found it necessary to stabilize the gapped duplex with $\mathrm{T}_{4}$ gene 32 protein (a single strand binding protein) during isolation and purification from the gel. Without this step we were unable to recover the gapped duplex as a circle on a preparative scale. The single stranded PCR products needed a slight modification at their $3^{\prime}$ end, because as determined by the acrylamide gel, one of the products contained at least one extra nucleotide. We "repaired" these ends with $\mathrm{T}_{4}$ polymerase, using it first as an exoruclease and then as a polymerase. For more aetail, see the accompanying photo copy of our short report.

After filling the gapped duplex with one of the single strand PCR products, the preparations were ligated and the supercoiled product was isolated from a gel. The assistant who worked with this project ended her stay in this country just after producing the supercoiled product. As soon as I have an available pair of hands we will reproduce these results and 
transfect cells with the DNA.

\section{Can We Identify Separate Mismatch Repair Systems Operating in Mammalian Colls?}

Over the course of this project we have noted that experimental variation between independent transfection experiments occurred that could not be assigned to the transfecting DNA's. During the last granting period we have been able to define the sources of some of this variation.

Because mismatches in DNA arise from different causes, correction to the original base pair must rely on different signals to identify the correct base in the mispair. We have theorized that different repair systems could be active in different compartments of the cell cycle. For instance, a system capable of recognizing replication errors would be expected to be active during the DNA synthesis phase (S phase). Such a system might be expected to recognize nicks as indicators of which strand is newly synthesized, and therefore, the appropriate one to repair. Alternatively, a repair system which reacts specifically to mismatches arising during interphase may be less dependent on nicks, especially distant nicks.

Our approach was to attempt to alter the strand bias of repair by controlling cell cycle. Our first successful attempts in this regard used a very simple approach. We transfected cells that were either actively growing or were stationary. We divided actively growing into subcategories based on how long the culture could be expected to continue active growth. This was measured as degree of confluency. When we transfected these cultures of cells with identical quantities of a heteroduplex DNA which contained a pair of nicks on alternate stranos, we found a difference in repair between these cultures. Cultures of cells in rapid growth were more influenced by the nicks than cultures that were at or near confluency.

Because the rapidly growing cultures had approximately twice the number of cells as the confluent cultures, we also tested whether the amount of DNA transfected into cells affected repair bias. We tested the highest and lowest confluency levels for their response to differing quantities of the heteroduplex DNA. In both cases increasing levels of transfecting DNA had the effect of decreasing the response to the signal of the nicks without affecting the percentage of DNA repaired. However, the amount of DNA required to shift the responses was perhaps ten 
times greater for the non-growing cells as the actively growing cells. (Data not shown but represents restriction analysis of greater than 400 plaques.)

The chief characteristic of growing cells is that they spend approximately one half of their time in $S$ phase. Stationary cells, however, are blocked in a $G_{0}$ phase from which they require an environmental change to emerge. Because we do not know at what time following transfection mismatch repair occurs, it becomes difficult to identify the precise effect of cell cycle on repair. However, actively growing cells are more likely to be in $S$ phase at any given time than stationary cells or cells approaching stationary phase. The difference in response we have seen leads us to believe that $S$ phase cells use a repair system which is dependent on nicks for its primary signals and that interphase cells are less dependent on these signals.

My next step will be to identify the time following transfection at which repair occurs. The time of repair may simply depend on the time required for the DNA to traverse from the exterior of the cell to the nucleus and be recognized by host cell enzymes or it may additionally require the cell to be stimulated (by SV40 Tantigen, for instance) to enter a specific portion of the cell cycle. Our plan is to transfect cells with a heteroduplex that has a decided repair bias and take samples over the next 48 hours which will then be amplified by PCR, followed by restriction analysis. The restriction pattern should change from an equal mixture of genotypes before repair to predominantly one genotype after repair. Similar experiments much earlier using a Southern blot were inconclusive but our expectation is that PCR will give us clearer results.

We have also been working on identifying which DNA polymerase is involved in this repair - one of the chromosome replication enzymes $\alpha$ or or, $\beta$ the so-called repair polymerase. In vitro work has identified $\alpha$ in one in vitro mammalian system and $\beta$ was identified by a second group in, what must presumably be, a second mammalian system. Aphidicolin and dideoxythymidine differentially inhibit $\alpha$ and $\beta$ DNA polymerases. We treated cells either before or after transfection with heteroduplex DNA. Aphidicolin completely blocked growth in these cells. These cultures ceased to divide. In all but one trial with this inhibitor no 
plaques resulted. The implication is that if viral reproduction is inhibited in the first 50 hours following transfection subsequent growth of the virus is not possible. Cells grew normally, however, in dideoxythymidine, even at $1 \mathrm{mM}$. We were initially concerned that this inhibitor might not be eniering the cell. I, therefore, tried adding fluorodeoxyuridine which does improve the uptake of thymidine. Although fluordeoxyuridine alone blocked cell growth as effectively as Aphidicolin, supplying dideoxythymidine along with fluorodeoxyuridine relieved the block, indicating that in this case, dideoxythymidine is entering the cell. An interesting sideline is that dideoxythymidine must be able to substitute for thymidine in this situation.

Of all the treatments the only one which produced a change in the bias was the combination of dideoxythymidine in the present of fluorodeoxyuridine administered for 50 hours after the transfection. Without inhibitor treatment the supercoiled heteroduplex is repaired in slightly more than three quarters of the molecules to restore a $\mathrm{G}: \mathrm{C}$ base pair and in about one quarter to A:T. This may reflect the near perfect repair of all G:T's to G:C and a random repair of $A: C$ 's to either $G: C$ or $A: T$.

Such an interpretation is consistent with all our data and with the data of others. The specific G:T repair in vitro appears to be the result of $\beta$-polymerase based on the ability of dideoxythymidine to suppress it, while non-specific repair in vitro appears to involve the use of $\alpha$ polymerase because Aphidicolin, but not dideoxythymidine, inhibits. Upon treatment of our cells with dideoxythymidine plus fluorodeoxyuridine, 10 to $30 \%$ fewer molecules were repaired to $\mathrm{G}: \mathrm{C}$. This would be expected to occur if a specific $\mathrm{G}: \mathrm{T} \rightarrow \mathrm{G}: \mathrm{C}$ repair reaction were being partially but not completely inhibited.

We intend to follow up these experiments with similar ones to compile enough data on these treatments to make a stronger case. If we are able to narrow the time interval in which we can be confident that mismatch repair is occurring then we may be able to confine treatments to shorter time periods and possibly increase the recovery of plaques in these experiments. 
This area of our work is perhaps the most enticing. Certainly there are multiple repair systems operative in mammalian cells. If we can find ways to isolate these systems in vivo either by cell cycle compartmentation or inhibitors, then we will have a system analogous to mutants in bacteria.

The last several months work has led us to an exciting point in at least three areas of our research. All of this work has been done with very limited personnel. A master's student, Brian Freeman did some of the initial work on competing nicks in heteroduplexes; Ina Babilon-Hesse, a master's level research assistant paid by the grant produced the separate $G / C$ and $A / T$ mismatches and the author of this report and co-principal investigator, Joan Hare, did all the remainder of the work. We thank the Department of Energy for its support of this project and look forward to finding future support for the project. 
$\because 2+\ldots$.

)

FIGURE 2 - SV40 heteroduplexes were formed by reannealing denatured DNA's of two genotypes which differed at only one base pair. Each heteroduplex carried two separate nicks which were on opposite strands. A heteroduplex pair was constituted of the two alternative pairings of nick to DNA genotype. CV-1 (African Green Monkey kidney) cells were transfected with either of the members of the heteroduplex pair and the resulting progeny plaques were analyzed by PCR plus restriction digest to determine which genotype it contained. Bias ratio was the number of plaques of one genotype divided by the total number of plaques of a single genotype produced by that heteroduplex. The difference between the bias ratios of a pair of nicked heteroduplexes is equal to the proportion of plaques repaired to $A: T$ when the closer nick was in the $C: G$ genotype strand minus the proportion of plaques repaired to $A: T$ when the closer nick was in the A:T genotype strand. T'is difference is an indicator of whether a particular nick has an effect on the repair bias. If switching the nick to the strand with the alternate genotype does not alter the bias ratio, then the nick is presumed to have no effect. Alternatively, any positive difference in the bias ratio indicates an effect from the nick. A positive difference indicates that the closer nick tended to increase the removal of the strand it was in. 

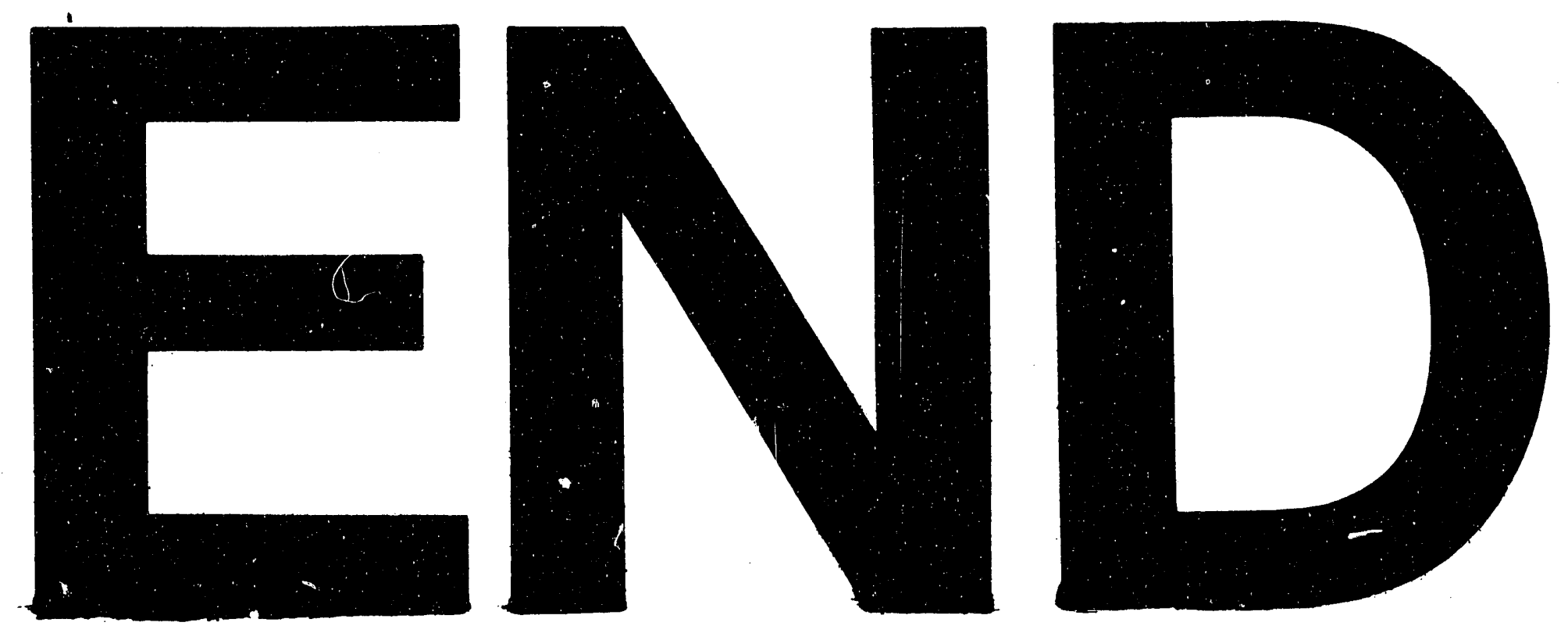

$x=1$

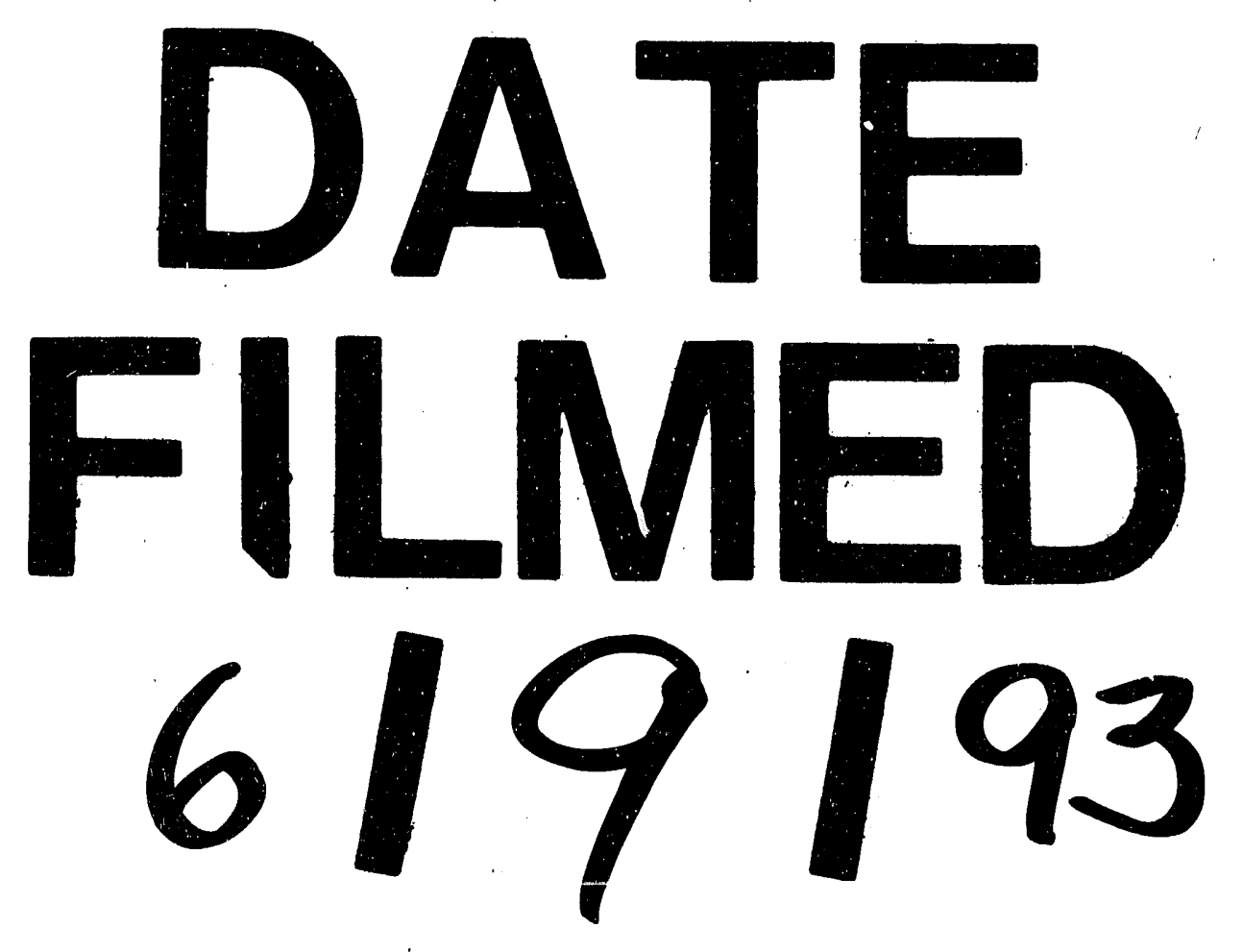


